\title{
Clinical outcomes of dialysis patients with COVID-19 in the initial phase of the COVID-19 outbreak in Wuhan, China
}

\author{
Xingruo Zeng ${ }^{1}$ - Xiaomei Huang ${ }^{1} \cdot$ Li Xu$^{1}$ - Jianwei Xiao ${ }^{2}$. Lianqing $\mathrm{Gu}^{1} \cdot$ Yin Wang $^{1} \cdot$ Yanhong Tuo $^{1} \cdot$ Xun Fang $^{1}$.

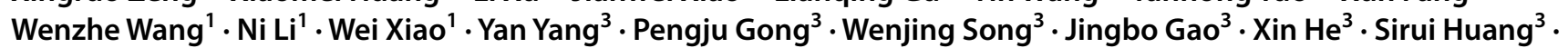 \\ Yifan Zeng ${ }^{3} \cdot$ Lei Wei $^{3} \cdot$ Wenli Chen ${ }^{1}$
}

Received: 28 April 2020 / Accepted: 28 September 2020 / Published online: 29 October 2020

(c) Springer Nature B.V. 2020

\begin{abstract}
Purpose Since the end of 2019, dialysis patients have been at risk of coronavirus disease 2019 (COVID-19) as well as other potential complications. Hence, we sought to describe the clinical characteristics of dialysis patients with severe acute respiratory syndrome coronavirus 2 (SARS-CoV-2) infection.

Methods We reviewed clinical outcomes, which consisted of clinical data extracted from the medical records of 695 registered dialysis patients at the Dialysis Center of Central Hospital of Wuhan from January 13th, 2020, to February 29th, 2020, and performed statistical analysis. According to the results, there were 447, 227 and 21 hemodialysis (HD), peritoneal dialysis (PD) and combined HD and PD (HD\&PD) cases, respectively.

Results During the outbreak of COVID-19, 36 dialysis patients were infected by SARS-CoV-2. Among those 36 patients, $32(7.2 \%)$ were on HD, and 4 (1.8\%) were on PD. When comparing SARS-CoV-2 infection between HD and PD, the relative risk was $4.07(\mathrm{RR}=4.07,95 \% \mathrm{CI} 1.46-11.35)$. We noted a median age of 66 years during the observation period, and the number of male patients was 23 (63.9\%). There were 15 fatal cases tested positive for SARS-CoV-2 (13 cases on HD and 2 cases on PD). By comparing mortality in the same period of 2018, 2019 and 2020, the all-cause mortality of hemodialysis patients was significantly higher in 2020 (4.89\%) than in either 2018 (2.55\%) or 2019 (1.97\%). There was no significant difference in mortality from all causes excluding COVID-19, during the same period among the 3-year period. However, during the COVID-19 outbreak, the mortality from all causes excluding COVID-19 was $2.73 \%$, which was slightly higher than that from COVID-19 (2.16\%).

Conclusions Although COVID-19 seriously threatens the health of people with uremia, deaths from all causes excluding COVID-19 during the epidemic cannot be ignored.
\end{abstract}

Keywords Hemodialysis $\cdot$ Peritoneal dialysis $\cdot$ COVID-19 $\cdot$ Clinical outcome $\cdot$ Uremia

Xingruo Zeng, Xiaomei Huang, and Li Xu have contributed equally.

Electronic supplementary material The online version of this article (https://doi.org/10.1007/s11255-020-02670-0) contains supplementary material, which is available to authorized users.

Lei Wei

leiwei@whu.edu.cn

Wenli Chen

bicwl@163.com

Extended author information available on the last page of the article

\section{Introduction}

In late December 2019, atypical pneumonia cases caused by severe acute respiratory syndrome coronavirus 2 (SARS-CoV-2) started being reported in Wuhan [1]. This novel virus soon spread widely among the city of 11 million populations [2,3]. In the early phase of transmission, the healthcare system in the city was strongly overwhelmed by the surge of patients with coronavirus disease 2019 (COVID-19). To our knowledge, there were no outcome data available for a unique group of patients on regular maintenance dialysis during this early phase of the outbreak. To describe the clinical characteristics of these patients and determine whether they represent 
a distinct patient group, we retrospectively analyzed the outcomes of 695 registered maintenance dialysis patients between January 13th, 2020, and February 29th, 2020. Among these registered patients, there were 447 on maintenance hemodialysis (HD), 227 on maintenance peritoneal dialysis (PD) and 21 on the combination of the two (HD\&PD).

\section{Materials and method}

\section{Study design, participants and data collection}

This observational study carried out at the Dialysis Center of Central Hospital of Wuhan (DC-CHWH), a tertiary teaching hospital that carries the second largest dialysis patient volume in the city. The observation period lasted nearly 60 days, from January 13th, 2020 to February 29th, 2020. According to the New Coronavirus Pneumonia Prevention and Control Program (7th edition) published by the National Health Commission of China [4], we retrospectively analyzed the clinical outcomes of 695 patients, who underwent maintenance dialysis in the early phase of COVID-19 outbreak. The analysis showed that 36 patients were diagnosed with COVID-19 based on their exposure history, clinical symptoms and related examinations, including laboratory examination and imaging examination.

\section{Real-time reverse transcription-polymerase chain reaction for SARS-CoV-2}

First, we extracted the respiration with an RNA isolation kit (Beijing Tiangen Biochemical Technology), after taking samples of human throat swabs and alveolar lavage fluid from patients suspected of having SARS-CoV-2 infection. Then, the SARS-CoV-2 nucleic acid detection kit (Wuhan Huada Biological Technology Co., Ltd.) was used for real-time RT-PCR to detect the content of SARS$\mathrm{CoV}-2$ in the sample.

\section{Statistical analysis}

Categorical variables were expressed as the frequency rate and the percentage, and continuous variables were represented using mean. Mean and median for continuous variables were compared using independent group $t$-tests when all data were normally distributed; otherwise, the Mann-Whitney $U$ test was used. Data (non-normal distribution) from repeated measures were compared using the generalized linear mixed model. Proportions for categorical variables were compared using the $\chi^{2}$ test while the Fisher exact test was used when encountering limited data. All statistical analyses were performed using SPSS 23.0. All tests were two-sided with significance level at $\mathrm{p}$ less than 0.05 .

\section{Results}

\section{Timeline of patient diagnosis and infection prevention measures}

There were $15 \mathrm{HD}$ patients and $2 \mathrm{PD}$ patients with confirmed COVID-19 in January and 17 HD patients and 2 PD patients in February (Fig. 1).

\section{Baseline characteristics of patients on maintenance dialysis}

The baseline characteristics and demographics of the patients and their baseline laboratory results while on dialysis are displayed in Table 1. Based on comparison of the two types of dialysis (HD and PD) with regard to age and length, HD patients were older and had longer dialysis times compared to PD patients.

\section{Clinical characteristics of dialysis patients with COVID-19}

Compared to patients on PD, patients on HD had much higher rates of COVID-19 infection, with rates of 7.2\% for HD and $1.8 \%$ for PD (RR $=4.07,95 \%$ CI 1.46-11.35). All



Fig. 1 Timeline of patient diagnosis and infection prevention measures 
Table 1 Baseline characteristics of maintenance dialysis patients

\begin{tabular}{llllll}
\hline Clinical characteristics & HD & PD & HD\&PD & $P^{\mathrm{a}}$ & $P^{\mathrm{b}}$ \\
\hline Number of cases & 447 & 227 & 21 & & \\
Age, years & $64(55-71)$ & $61(50-68)$ & $63.00(43.50-65)$ & 0.001 & $<0.001$ \\
Age groups & & & & 0.076 & 0.07 \\
$\quad<65$ years & $241(53.9)$ & $139(61.2)$ & $15(71.4)$ & & \\
$\geq 65$ years & $206(46.1)$ & $88(38.8)$ & $6(28.6)$ & & \\
Sex & & & & & \\
Female & $173(38.7)$ & $104(45.8)$ & $5(23.8)$ & & \\
$\quad$ Male & $274(61.3)$ & $123(54.2)$ & $16(76.2)$ & & \\
Dialysis session length, m & $30(18-48)$ & $24(12-36)$ & $38(23-61)$ & & \\
Underline disease & & & & & \\
$\quad$ Diabetes & $184(41.2)$ & $66(29.1)$ & $8(38.1)$ & 0.0016 & $<0.001$ \\
Hypertension & $409(91.5)$ & $205(90.3)$ & $21(100.0)$ & 0.002 \\
Cardiac disease & $423(94.6)$ & $211(93.0)$ & $21(100.0)$ & 0.418 & 0.383 \\
Respiratory disease & $61(13.7)$ & $19(8.4)$ & $2(9.5)$ & 0.12 & 0.045 \\
Laboratory tests & & & & & \\
Hb, g/L & $101.6 \pm 18.6$ & $98.7 \pm 19.7$ & $96.8 \pm 15.1$ & 0.092 & 0.065 \\
Alb, g/L & $40.1(36.5-42.8)$ & $34.65(31.1-37.8)$ & $39.40(35.7-43.8)$ & $<0.001$ & 0.004 \\
Blood phosphorus, mmol/L & $1.6(1.3-2.0)$ & $1.51(1.3-1.8)$ & $1.5(1.2-2.0)$ & 0.007 & 0.003 \\
iPTH, pmol/L & $29.8(14.9-51.0)$ & $22.38(10.8-42.2)$ & $24.03(8.2-52.8)$ & 0.004 & 0.006 \\
\hline
\end{tabular}

${ }^{\mathrm{a}} p$ value by ANOVA, Kruskal-Wallis test and Chi-square test among HD, PD and HD\&PD group;

${ }^{\mathrm{b}} p$ value by $t$-test, Mann-Whitney $U$ test and Chi-square test between HD and PD group. Continuous variables were shown as Mean \pm SD when the data were normally distributed; otherwise, continuous variables were shown as median (interquartile range) these differences were statistically significant (Table 1). The clinical characteristics of dialysis patients with COVID-19 are shown in Table S1. During the outbreak of COVID-19, 36 dialysis patients were infected by the virus. The median age was 66 years, and the number of male patients was 23 (63.9\%). Common symptoms at the onset of illness included fever (77.8\%), cough $(55.6 \%)$ and fatigue $(36.1 \%)$. Most patients had abnormal laboratory results, including lymphopenia $(77.8 \%)$, anemia $(41.7 \%)$ and elevated C-reactive protein (80.6\%). As of February 14 th, 2020, we began to periodically track hematological and biochemical parameters of 12 hospitalized HD patients at day 1 , day 4 , day 7 and day 14 after disease onset. During this period, lymphopenia was diagnosed in most cases. As the disease progressed, the white blood cell count was higher in nonsurvivors than in survivors, while the neutrophil count was similar between the two groups. However, the level of hemoglobin in nonsurvivors was higher than that in survivors, though this was not statistically significant (Figure S1). All patients (100\%) presented with characteristic ground-glass opacities on chest computed tomographic scans, $80.9 \%$ of them had multifocal involvement, $66.7 \%$ of them had pleural effusion or pericardial effusion, and $44.4 \%$ of them had consolidation and atelectasis (Table S1).

\section{Mortality in maintenance dialysis patients}

During the observation period, 34 dialysis patients died. Among these cases, 15 of the patients died of COVID-19, while 19 died from causes other than COVID-19 as shown in Table S2. By comparing the mortality in the same period of 2018, 2019 and 2020, the all-cause mortality of hemodialysis patients was significantly higher in $2020(4.89 \%)$ than in $2018(2.55 \%)$ or 2019 (1.97\%) (Table 2). With no significant difference, mortality rates from all causes excluding COVID-19 during the same period among the 3 years were $2.55 \%$ in $2018,1.97 \%$ in 2019 , and $2.73 \%$ in 2020 . During the COVID-19 outbreak, the mortality from all causes

Table 2 The mortality of maintenance dialysis patients in the same period in 2018, 2019, 2020

\begin{tabular}{llllll}
\hline The cause of death & 2018 & 2019 & 2020 & $P^{\mathrm{a}}$ & $P^{\mathrm{b}}$ \\
\hline COVID-19 & 0 & 0 & 2.16 & & \\
$\begin{array}{l}\text { The causes excluding } \\
\text { COVID-19 }\end{array}$ & 2.55 & 1.97 & 2.73 & 0.835 & 0.535 \\
\begin{tabular}{l} 
All-cause \\
\hline
\end{tabular} & 2.55 & 1.97 & 4.89 & 0.029 & 0.003 \\
\hline
\end{tabular}

${ }^{\text {a }}$ The mortality in 2020 compared with that in 2018

${ }^{\text {b }}$ The mortality in 2020 compared with that in 2019 
excluding COVID-19 was $2.73 \%$, which was slightly higher than that from COVID-19 (2.16\%) (Table 2).

\section{Discussion}

In December 2019, SARS-CoV-2 caused a major outbreak of respiratory infectious disease in Wuhan. Epidemiological investigations have shown that the elderly or patients with chronic diseases are more susceptible to COVID-19 [4, 5]. As dialysis patients have poor physical fitness, they are likely to be susceptible to infectious diseases [6].

The results of this study indicate that HD patients were more likely than PD patients to be infected with SARSCoV-2 in our hospital ( $R R=4.07,95 \%$; CI: $1.46-11.35$ ). We speculate that patients on PD had less hospital exposure than did patients on $\mathrm{HD}$, which may reduce their risk of contracting COVID-19 during this early period of the COVID-19 outbreak. Furthermore, PD patients and their families practiced a routine that required them to wear masks and use hand hygiene when performing PD operations according to the Standard Operating Procedure, [7] which may objectively block the transmission of droplets or contact transmission risk. In addition, ultraviolet radiation was also routinely applied to disinfect materials and the environment around PD patients. In contrast, there was no such compulsory requirement for HD patients at home [8]. On January $13^{\text {th }}$, after the first HD patient was diagnosed with COVID-19, emergency infection prevention measures were implemented in our hemodialysis unit. In addition to vigorous environmental cleaning, these infection prevention measures included but were not limited to prescreening all patients and visitors on arrival at the hospital, offering face masks and hand hygiene, designating clinical times for dialysis patients and isolating dialysis patients from other patient populations. As a result, none of the 21 HD\&PD patients were infected with SARS-CoV-2, which further strengthened the importance of wearing masks and hand hygiene and disinfection as self-protection during the COVID-19 epidemic. Furthermore, the citywide lockdown on January 23th significantly decreased the risk of cross infections among the general population, emphasizing the efficacies of home isolation and infection prevention measures in the hemodialysis unit. It also has a significant effect on guiding the management process and detailed rules of dialysis centers in the future.

The most common symptoms at the onset of illness we observed were fever cough, fatigue, lymphopenia, elevated levels of C-reactive protein and abnormal chest computed tomographic scans with ground-glass opacities. These clinical characteristics of the onset of illness were consistent with the latest studies [5, 9-11]. It is worth noting that more than half of the HD patients had pleural effusion or pericardial effusion, which may be related to uremia.
The unexpectedly increased mortality rate during the COVID-19 epidemic is being caused by COVID-19 infection, and the infection seriously threatens the health of people with uremia. However, during the COVID-19 epidemic, deaths from all causes excluding COVID-19 cannot be ignored. Conventional treatments such as regular dialyses are still vital for dialysis patients. Therefore, it is not wise to discontinue dialysis treatments, even during the COVID-19 outbreak, since dialysis patients are at high risk for serious complications and even death.

The publication by the Columbia University Vagelos College of Physicians Working Group describes the principal elements of their response, early lessons learned during the pandemic in New York City and the major reconfigurations of services [12]. Similarly, for dialysis patients infected with SARS-CoV-2, we have taken sufficient protective measures, such as isolation measures, separate dialysis machines and dialysis nurses. However, we focused on different populations. Furthermore, we only statistically analyzed patients with maintenance dialysis, whereas patients with acute kidney injury or kidney transplants were not included in our study.

In summary, patients on hemodialysis have a higher risk of SARS-CoV-2 infection than do patients on peritoneal dialysis. We suggest that special protections, including screening close contacts to minimize potential viral exposure, are required. COVID-19 seriously threatens the health of people with uremia, though death from all causes excluding COVID-19 cannot be ignored during the COVID-19 epidemic. Therefore, it is not wise to discontinue dialysis treatments, even during the COVID-19 outbreak, since dialysis patients are at high risk for serious complications and even death.

Acknowledgements We thank all the medical, nursing, and technical staff from the Department of Nephrology, Central Hospital of Wuhan, for their dedicated care of our dialysis patient during the COVID-19 epidemic. We also thank all the authorized COVID-19 Dialysis Units for their help.

Author contributions Wenli Chen and Lei Wei designed the study; Dr. Chen and Prof. Wei had full access to all of the data in the study and take responsibility for the integrity of the data and the accuracy of the data analysis; Dr. Zeng, Huang and Xu contributed equally and share the first authorship; Jianwei Xiao is responsible for reviewing CT images; Pengju Gong, Jingbo Gao and Sirui Huang analyzed the data and made the figures; Yan Yang, Yifan Zeng and Xin He drafted the paper; Wenjing Song revised the paper; all authors approved the final version of the manuscript.

\section{Compliance with ethical standards}

Conflict of interest We declare no competing interests. 


\section{References}

1. Lu H, Stratton CW, Tang YW (2020) Outbreak of pneumonia of unknown aetiology in Wuhan, China: the mystery and the miracle. J Med Virol 92(4):401-402. https://doi.org/10.1002/jmv.25678

2. Chan JF, Yuan S, Kok KH, To KK, Chu H, Yang J, Xing F, Liu J, Yip CC, Poon RW, Tsoi HW, Lo SK, Chan KH, Poon VK, Chan WM, Ip JD, Cai JP, Cheng VC, Chen H, Hui CK, Yuen KY (2020) A familial cluster of pneumonia associated with the 2019 novel coronavirus indicating person-to-person transmission: a study of a family cluster. Lancet 395(10223):514-523. https:// doi.org/10.1016/S0140-6736(20)30154-9

3. Zhu N, Zhang D, Wang W, Li X, Yang B, Song J, Zhao X, Huang B, Shi W, Lu R, Niu P, Zhan F, Ma X, Wang D, Xu W, Wu G, Gao GF, Tan W, China Novel Coronavirus I, Research T (2020) A novel coronavirus from patients with pneumonia in China. N Engl J Med 382(8):727-733. https://doi.org/10.1056/NEJMoa2001017

4. National Health Commission of China (2020) New coronavirus pneumonia prevention and control program, 7th edn. http://www. gov.cn/zhengce/zheng ceku/2020-03/04/5486705/files/ae610 04f930d47598711a0d4cbf874a9.pdf. Accessed 3 Mar 2020 (in Chinese)

5. Yang X, Yu Y, Xu J, Shu H, Xia J, Liu H, Wu Y, Zhang L, Yu Z, Fang M, Yu T, Wang Y, Pan S, Zou X, Yuan S, Shang Y (2020) Clinical course and outcomes of critically ill patients with SARSCoV-2 pneumonia in Wuhan, China: a single-centered, retrospective, observational study. The Lancet Respiratory medicine. https ://doi.org/10.1016/S2213-2600(20)30079-5

6. Assiri A, McGeer A, Perl TM, Price CS, Al Rabeeah AA, Cummings DA, Alabdullatif ZN, Assad M, Almulhim A, Makhdoom H, Madani H, Alhakeem R, Al-Tawfiq JA, Cotten M, Watson SJ, Kellam P, Zumla AI, Memish ZA, Team KM-CI (2013) Hospital outbreak of Middle East respiratory syndrome coronavirus.
N Engl J Med 369(5):407-416. https://doi.org/10.1056/NEJMo a1306742

7. Chen XM (2010) Peritoneal dialysis standard operating procedure (SOP). Military Science Publishing House, Beijing, China

8. System CNRD (2019) Blood Purification Standard Operating Procedure (SOP) (The 2019 solicitation edition).

9. Pan Y, Guan H, Zhou S, Wang Y, Li Q, Zhu T, Hu Q, Xia L (2020) Initial CT findings and temporal changes in patients with the novel coronavirus pneumonia (2019-nCoV): a study of 63 patients in Wuhan, China. Eur Radiol. https://doi.org/10.1007/s00330-02006731-x

10. Wang D, Hu B, Hu C, Zhu F, Liu X, Zhang J, Wang B, Xiang H, Cheng Z, Xiong Y, Zhao Y, Li Y, Wang X, Peng Z (2020) Clinical characteristics of 138 hospitalized patients with 2019 novel coronavirus-infected pneumonia in Wuhan, China. JAMA. https ://doi.org/10.1001/jama.2020.1585

11. Xu X, Yu C, Qu J, Zhang L, Jiang S, Huang D, Chen B, Zhang Z, Guan W, Ling Z, Jiang R, Hu T, Ding Y, Lin L, Gan Q, Luo L, Tang X, Liu J (2020) Imaging and clinical features of patients with 2019 novel coronavirus SARS-CoV-2. Eur J Nucl Med Mol Imaging 47(5):1275-1280. https://doi.org/10.1007/s00259-02004735-9

12. Division of Nephrology, Columbia University Vagelos College of Physicians. Disaster Response to the COVID-19 Pandemic for Patients with Kidney Disease in New York City. J Am Soc Nephrol. 2020; 31(7):1371-1379. doi:https://doi.org/10.1681/ ASN.2020040520

Publisher's Note Springer Nature remains neutral with regard to jurisdictional claims in published maps and institutional affiliations.

\section{Affiliations}

\section{Xingruo Zeng ${ }^{1}$. Xiaomei Huang ${ }^{1} \cdot$ Li Xu$^{1}$. Jianwei Xiao ${ }^{2}$. Lianqing Gu ${ }^{1} \cdot$ Yin Wang $^{1}$ - Yanhong Tuo ${ }^{1} \cdot$ Xun Fang $^{1}$.

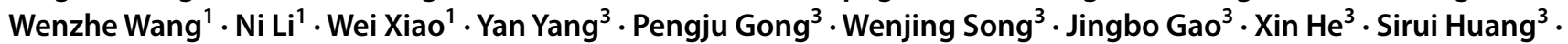 Yifan Zeng ${ }^{3} \cdot$ Lei Wei $^{3} \cdot$ Wenli Chen ${ }^{1}$}

Wei Xiao

Shincuwin@126.com

1 Department of Nephrology, The Central Hospital of Wuhan, Tongji Medical College, Huazhong University of Science and Technology, Wuhan 430014, Hubei, China
2 Department of Medical Imaging, The Central Hospital of Wuhan, Tongii Medical College, Huazhong University of Science and Technology Wuhan, Hubei 430014, China

3 Department of Pathology and Pathophysiology, School of Basic Medical Sciences, Zhongnan Hospital, Wuhan University, Wuhan 430071, Hubei, China 